\title{
Parabolic Methods for One Dimensional Advection-Diffusion Type Equation and Application to Burger Equation
}

\author{
Kedir Aliyi Koroche \\ Department of Mathematics, College of Natural and Computational Sciences, Ambo University, Ambo, Ethiopia \\ Email address: \\ kediraliyi39@gmail.com \\ To cite this article: \\ Kedir Aliyi Koroche. Parabolic Methods for One Dimensional Advection-Diffusion Type Equation and Application to Burger Equation. \\ International Journal of Applied Mathematics and Theoretical Physics. Vol. 7, No. 2, 2021, pp. 40-52. doi: 10.11648/j.ijamtp.20210702.11
}

Received: March 26, 2021; Accepted: May 21, 2021; Published: May 27, 2021

\begin{abstract}
In this paper, the piecewise parabolic method is presented for solving the one-dimensional advection-diffusion type equation and its application to the burger equation. First, the given solution domain is discretized by using a uniform Discretization grid point. Next by applying the integration in terms of spatial variable, we discretized the given advectiondiffusion type equation and converting it into the system of first-order ordinary differential equation in terms of temporarily variable. Next, by using Taylor series expansion we discretized the obtained system of ordinary differential equation and obtain the central finite difference equation. Then using this difference equation, the given advection-diffusion type equation is solved by using the parabolic method at each specified grid point. To validate the applicability of the proposed method, four model examples are considered and solved at each specific grid point on its solution domain. The stability and convergent analysis of the present method is worked by supported the theoretical and mathematical statements and the accuracy of the solution is obtained. The accuracy of the present method has been shown in the sense of root mean square error norm $\mathrm{L}_{2}$ and maximum absolute error norm $\mathrm{L}_{\infty}$ and the local behavior of the solution is captured exactly. Numerical, exact solutions and behavior of maximum absolute error between them have been presented in terms of graphs and the corresponding root means square error norm $\mathrm{L}_{2}$ and maximum absolute error norm $\mathrm{L}_{\infty}$ presented in tables. The present method approximates the exact solution very well and it is quite efficient and practically well suited for solving advection-diffusion type equation. The numerical result presented in tables and graphs indicates that the approximate solution is in good agreement with the exact solution. Hence the proposed method is accruable to solve the advection-diffusion type equation.
\end{abstract}

Keywords: Advection-diffusion Type Equation, Burger Equation, Piece-wise Parabolic Method, Taylor Series Expansion, Stability, Convergent Analysis, Root Mean Square and Maximum Absolute Error Norm

\section{Introduction}

The nonlinear advection-diffusion type equation is one of the popular and important models describing many phenomena derived from various areas of mathematical physics and engineering fields [1]. The nonlinear model arises in gas dynamics, water waves, electrodynamics, chemical reactions, transport of pollutants flood, and ecological systems [10]. This equation is also found in the form of hydrodynamics, shock waves, heat conduction [1], and it is called quasi-linear parabolic partial differential $[2,4$, 6]. The nonlinear homogenous quasi-linear parabolic partial differential is encountered in the theory of shock waves, mathematical modeling of turbulent fluid, and in continuous stochastic processes $[2,6]$. Such a type of equation was firstly introduced by Bateman [4] in 1915 and he proposed the steady-state solution of the problem [2]. In 1948, Burgers $[2,5,7]$ introduced this equation to capture some features of turbulent fluid in a channel caused by the interaction of the opposite effects of convection and diffusion [2, 21]. These types of the equation represent the Burgers equation, the heat conduction equation, the nonlinear Schrödinger equation, the Navier-Stokes equation [1]. Burgers' equation arises in many physical problems including one-dimensional turbulence, sound waves in a viscous medium, shock waves in a viscous medium, waves in fluid-filled viscous elastic tubes, and 
magnetohydrodynamic waves in a medium with finite electrical conductivity $[3,6]$

Nonlinear phenomena play a crucial role in applied mathematics and physics [17]. The importance of obtaining the exact or approximate solutions of PDEs in physics and mathematics is still a hot topic as regards seeking new methods for obtaining new exact or approximate solutions [3, $8,13,15,17]$. For that purpose, different methods have been put forward for seeking various exact solutions of multifarious physical models described using nonlinear PDEs [17]. A well-known model was first introduced by Bateman [4], who found its steady solutions, descriptive of certain viscous flows. It was later proposed by Burgers [14] as one of a class of equations describing mathematical models of turbulence [17]. Khater et al. [12] proposed the Chebyshev spectral collocation method for solving the coupled Burgers' equations [31]. With pseudo-spectral methods care must be taken with the round-off error issue when higher derivatives or a large several points $N$ is involved [12]. In their work Gowrisankar, S. and Natesan, S. [16], present the numerical solution of singularly perturbed initial-boundary Burgers' equation by using an efficient robust numerical method. They provide an $e$-uniformly convergent numerical method for the singularly perturbed Burger [16]. They obtain uniform convergence concerning the perturbation parameter $\mathcal{E}$. Even though the method is capable of approximating Burger's equation, they failed to solve a relatively small perturbation parameter. Amit et al. [11] Present solution of burger equation using seventh order convergent weakly $L$-stable Newton Cotes formula and application of Burger's equation. They used Hermit's interpolation polynomial approximation (oscillatory interpolation) and explicit backward Taylor's polynomial approximation. Both Hermits interpolation and Taylor series method is computationally time-consuming for a large number of the grid point. So the solution is containing high contaminated round-off error and truncation error. Soyoon et al. [1] presented a solution of the nonlinear advection-diffusion equation by a backward semi-Lagrangian method. The method is computationally difficult when the number for a large number of the grid point. Sachin [10] presents the numerical solution of the burger equation by using the -Nicolson type method. This method is of computational cost because it requires a large number of grid points to give an accurate solution for the Burger equation [30]. Reza Abazaria and Borhanifar [17] presented the solution of Burgers and coupled Burgers' equation by using the differential transform method (DTM). DTM is a seminumerical-analytic technique that formalizes the Taylor series differently. The Taylor series method is computationally time-consuming for large orders and high contaminated round-off error and truncation error.

However, still, the accuracy of the method needs attention; because the treatment of the method used to solve the nonlinear advection-diffusion type equation is not trivial distribution. Even though the accuracy of the aforementioned methods needs attention, they require large memory and long computational time. So the treatments of this method present severe difficulties that have to be addressed to ensure the accuracy of the solution. To this end, this paper aims to develop a parabolic method that is capable of solving onedimensional advection-diffusion and approximate the exact solution. The convergence has been shown in the sense of $L_{\infty}$ norm and $L_{2}$ norm so that the local behavior of the solution is captured exactly. The stability and convergence of the present method are also investigated by using Von Neumann stability analysis techniques.

Statement of the problem

Consider that the following nonlinear advection-diffusion type equation considered in [1] given by:

$$
u_{t t}+u u_{x}=v u_{x x}(x, t) \in(a, b) \times(0, T)
$$

with initial and boundary condition respectively

$$
\begin{gathered}
u(x, 0)=f(x), a \leq x \leq b, \\
u(a, t)=g_{1}(t), u(b, t)=g_{2}(t), 0 \leq t \leq T
\end{gathered}
$$

where $v>0$ is the coefficient of kinematic viscosity and $\left.\mathrm{f}(\mathrm{x}), \mathrm{g}_{1}(\mathrm{t})\right)$ and $\mathrm{g}_{2}(\mathrm{t})$ assumed to be sufficiently smooth functions for the existence and the uniqueness of the solution $[18,19]$. The solution u may represent a temperature for heat transfer or a species concentration for mass transfer at position $\mathrm{x}$ and time $\mathrm{t}$ with the advection velocity $\mathrm{u}$ [1]. The local subdomain corresponding to each node can firstly be determined based on the Euclidean distance between the nodes [9].

Now we define a mesh size $\mathrm{h}$ and $\mathrm{k}$ and the constant grid point by drawing equidistance horizontal and vertical lines of distance ' $h$ ' and ' $k$ ' respectively in ' $x$ ' and ' $t$ ' direction. These lines are called gridlines and the point at which they interacting is known as the mesh point. The mesh point that lies at end of the domain is called the boundary point. The points that lie inside the region are called interiors points. The goal is to approximate the solution ' $\mathrm{u}_{\mathrm{j} n}$ ' at the interior mesh points. Hence we discretized the solution domain as:

$$
\begin{gathered}
a=x_{0}<x_{1}<x_{2}<\cdots<x_{M}=b \\
0=t_{0}<t_{1}<t_{2}<\cdots<t_{N}=T
\end{gathered}
$$

Where $\quad x_{j+1}=x_{j}+j h \quad$ and $t_{n+1}=t_{n}+n k \quad, \quad j=$ $0(1) M, n=0(1) N . M$ and $N$ are the maximum numbers of grid points respectively in the $\mathrm{x}$ and $\mathrm{t}$ direction. Then the present paper is organized as follows. Section two is a description of numerical methods, section three is Stability and convergence analysis, section four is Numerical results. Section five is Discussion; section six is the conclusion.

\section{Description of Numerical Methods}

In this paper, the parabolic method is developed to solve the nonlinear parabolic partial differential equation given in Eq. (1). These nonlinear parabolic partial differential equations such as nonlinear advection-diffusion type equation have first-order derivatives in time and first \& second order in space.

Parabolic Methods 
PPM is a third-order Godunov-type method [20]. This method has several remarkable features that distinguish it from other Godunov-type schemes [18]. The scheme is conservative, dimensionally UN split, and suitable for a general equation of state $[18,20]$. Temporal evolution is second-order accurate and employs characteristic projection operators; spatial interpolation is piece-wise parabolic making the scheme third-order accurate in smooth regions of the flow away from discontinuities [19]. Second, the representation of the nonlinear wave interactions used to calculate fluxes is greatly simplified, making the algorithm simpler and more robust $[18,19]$. The features make, PPM highly suitable for multi-fluid problems with complex structures such as shock waves and contact discontinuities [18]. Numerical tests prove that it outperforms other schemes

$$
\begin{gathered}
\int_{x_{j-\frac{1}{2}}}^{x_{j+\frac{1}{2}}} u_{t} d x-\int_{x_{j-\frac{1}{2}}}^{x_{j+\frac{1}{2}}} u_{x x} d x=-\int_{x_{j-\frac{1}{2}}}^{x_{j+\frac{1}{2}}}[\psi(u)]_{x} d x \\
\int_{x_{j-\frac{1}{2}}}^{x_{j+\frac{1}{2}}} u_{t} d x-v\left[u_{x}\right]_{x_{j-\frac{1}{2}}}^{x_{j+\frac{1}{2}}}=-[\psi(u)]_{x_{j-\frac{1}{2}}}^{x_{j+\frac{1}{2}}}
\end{gathered}
$$

Now from Eq. (6), we can assume that

$$
\begin{gathered}
\int_{x_{j-\frac{1}{2}}}^{x+\frac{1}{2}} u_{t} d x \approx \frac{d u\left(x_{j}, t\right)}{d t} \\
v\left[u_{x}\right]_{j-\frac{1}{2}}^{x_{j+\frac{1}{2}}} \approx-v\left[u_{x}\left(x_{j+\frac{1}{2}}, t\right)-u_{x}\left(x_{j-\frac{1}{2}}, t\right)\right]=-\frac{v}{h^{2}}\left[u\left(x_{j+1}, t\right)-2 u\left(x_{j}, t\right)+u\left(x_{j-1} t\right)\right] \\
-[\psi(u)]_{j-\frac{1}{2}}^{x_{j+\frac{1}{2}}} \approx \frac{1}{h}\left[\psi\left(u_{j-\frac{1}{2}}, t\right)-\psi\left(u_{j+\frac{1}{2}}, t\right)\right]
\end{gathered}
$$

Now By substituting Eq.(7) into Eq.(6) we obtain the system of the nonlinear ordinary differential equation given in the form:

$$
\frac{d u\left(x_{j}, t\right)}{d t}=\frac{v}{h^{2}}\left[u\left(x_{j+1}, t\right)-2 u\left(x_{j}, t\right)+u\left(x_{j-1} t\right)\right]+\frac{1}{h}\left[\psi\left(u_{j-\frac{1}{2}}, t\right)-\psi\left(u_{j+\frac{1}{2}}, t\right)\right]
$$

subjected to initial and boundary condition:

$$
\begin{gathered}
u\left(x_{j}, 0\right)=f\left(x_{j}\right), a \leq x_{j} \leq b, \\
u(a, t)=g_{1}(t), u(b, t)=g_{2}(t), 0 \leq t \leq T
\end{gathered}
$$

\section{Temporal Discretization}

Assuming that $u(x, t)$ has continuous higher order partial derivative on the region $[0, L] x[0, T]$. For the sake of simplicity, we use $u\left(x_{j}, t_{n}\right)=u_{j n}$ and $\frac{d^{p} u}{d t^{p}}=d_{t}^{p} u_{j n}$ for $p \geq 1$ is $p^{t h}$ order derivatives. By using Taylor series expansion, we have

$$
\begin{gathered}
u_{j n+1}=u_{j n}+k d_{t} u_{j n}+\frac{k^{2}}{2 !} d_{t}^{2} u_{j n}+\frac{k^{3}}{3 !} d_{t}^{3} u_{j n}+\ldots \\
u_{j n-1}=u_{j n}-k d_{t} u_{j n}+\frac{k^{2}}{2 !} d_{t}^{2} u_{j n}-\frac{k^{3}}{3 !} d_{t}^{3} u_{j n}+\cdots
\end{gathered}
$$

Subtract the second equation from the first equation we obtain the central difference equation of the first-order derivative of the function concerning temporal variable at the point $\left(x_{j}, t_{n}\right)$ :

$$
d_{t} u_{j n}=\frac{u_{j n+1}-u_{j n-1}}{2 k}+\tau_{1}
$$


Where $\tau_{1}=-\frac{k^{2}}{6} d_{t}^{3} u_{j n}$ is a local truncation error term. Now truncating the local truncation error and substituting Eq. (11) into Eq. (10) we obtain the full Discretization of governing partial differential equation with corresponding initial and boundary condition given in the form of

$$
\begin{gathered}
\frac{u_{j n+1}-u_{j n-1}}{2 k}=\frac{v}{h^{2}}\left[u\left(x_{j+1}, t_{n}\right)-2 u\left(x_{j}, t_{n}\right)+u\left(x_{j-1} t_{n}\right)\right]+\frac{1}{h}\left[\psi\left(u_{j-\frac{1}{2}}, t_{n}\right)-\psi\left(u_{j+\frac{1}{2}}, t_{n}\right)\right] \\
u_{j n+1}=u_{j n-1}+\alpha\left[u_{j+1, n}-2 u_{j, n}+u_{j-1, n}\right]+\beta\left[\psi_{j-\frac{1}{2}, n}-\psi_{j+\frac{1}{2}, n}\right]
\end{gathered}
$$

subjected to initial and boundary condition:

$$
\begin{gathered}
u\left(x_{j}, 0\right)=f\left(x_{j}\right), a \leq x_{j} \leq b, \\
u\left(a, t_{n}\right)=g_{1}\left(t_{n}\right), u\left(b, t_{n}\right)=g_{2}\left(t_{n}\right), 0 \leq t_{n} \leq T
\end{gathered}
$$

where $\quad \alpha=2 v k / h^{2} \quad, \quad \beta=\frac{2 k}{h}, u\left(x_{j}, t_{n}\right) \cong u_{j, n} \quad$ and $\psi\left(u_{j-\frac{1}{2}}, t_{n}\right) \cong \psi_{j-\frac{1}{2}, n}$ Now using the discrete scheme in Eqs. (12) and (13) we obtain the accurate numerical solution of the advection-diffusion type equation.

Stability and Convergent Analysis

The Fourier analysis (Von-Neumann) stability [32] analysis technique is applied to investigate the stability of the proposed method. Such an approach has been used by many researchers like [23-26, 32]. Now consider that the make nonlinearity in the difference scheme is linear by taking $\gamma=$ $\max u_{j}$ such that $\psi(u) \cong \frac{\gamma u}{2}$. Again assume that the trial solution of governing equation at $\left(x_{j}, t_{n}\right)$ is

$$
u(j, n)=\lambda^{n} e^{i j \theta}
$$

where $i=\sqrt{-1} \theta=\frac{a \pi}{M}, a \in \mathbb{R}$ and $\lambda$ is a complex number. Now using $\psi_{j-\frac{1}{2}, n} \approx \frac{\gamma u_{j-\frac{1}{2}, n}}{2}$ and substituting Eq. (14) into Eq.(13) we obtain:

$$
\begin{gathered}
\lambda^{n+1} e^{i j \theta}=\lambda^{n-1} e^{i j \theta}+\alpha\left[\lambda^{n} e^{i(j+1) \theta}-2 \lambda^{n} e^{i j \theta}+\right. \\
\left.\lambda^{n} e^{i(j-1) \theta}\right]+\beta\left[\lambda^{n} e^{i\left(j-\frac{1}{2}\right) \theta}-\lambda^{n} e^{i\left(j+\frac{1}{2}\right) \theta}\right] \\
\lambda=\lambda^{-1}+2 \alpha(\cos \theta-1)-2 \beta i \sin \left(\frac{\theta}{2}\right) \\
\lambda^{2}-\left[2 \alpha(\cos \theta-1)-2 \beta i \sin \left(\frac{\theta}{2}\right)\right] \lambda-1=0
\end{gathered}
$$

Let $X=\left(\alpha(\cos \theta-1)-\beta i \sin \left(\frac{\theta}{2}\right)\right)$, the above equation is rewritten as of the form:

$$
\lambda^{2}-2 X \lambda-1=0
$$

By using the Perfect square method the root of Eq. (15) is

$$
\begin{aligned}
& \lambda(a)=2 X \pm \sqrt{1-X^{2}} \quad \text { (16) of error is } \\
& \varepsilon_{j n+1}=\varepsilon_{j n-1}+\alpha\left[\varepsilon_{j+1, n}-2 \varepsilon_{j, n}+\varepsilon_{j-1, n}\right]+\beta\left[\varepsilon_{j-\frac{1}{2}, n}-\varepsilon_{j+\frac{1}{2}, n}\right] \\
& \alpha\left[\varepsilon_{j+1, n}-2 \varepsilon_{j, n}+\varepsilon_{j-1, n}\right]=\varepsilon_{j n+1}-\varepsilon_{j n-1}-\beta\left[\varepsilon_{j-\frac{1}{2}, n}-\varepsilon_{j+\frac{1}{2}, n}\right]
\end{aligned}
$$

Since $\alpha=2 v k / h^{2} \ll 1$ and $\beta=\frac{2 k}{h} \ll 1$ for $h \rightarrow 0$. Hence $|\lambda(a)|<1$. Thus this shows that the scheme is stable.

Definition [26]: A scheme considering being consistent with a partial differential equation if the truncation error associated with this scheme is tending to zero when the time and spatial step size tend to zero.

Definition [27]: The finite difference method is consistent in the $\|$.$\| -norm if the \lim _{\substack{h \rightarrow 0 \\ k \rightarrow 0}}\left\|\tau_{j n}\right\|=0$. Moreover, the method is the consistency of order $(p, q)$ if $\left\|\tau_{j n} \cdot\right\|=$ $O\left(h^{p}+k^{q}\right)$.

Now our proposed numerical scheme satisfies all of the above conditions. It means from Eq. (9) we have: $\tau_{1}=$ $-\frac{k^{2}}{6} d_{t}^{3} u_{j n}$ and $\tau_{2}=-\frac{h^{2}}{12} \partial_{x}^{4} u_{j n}$ are the local traction error associated with our proposed method.

$$
\begin{gathered}
\tau_{j n}=\left(\tau_{1}+\tau_{2}\right)=-\frac{k^{2}}{6} d_{t}^{3} u_{j n}-\frac{k v h^{2}}{6} \partial_{x}^{4} u_{j n} \\
\lim _{\substack{h \rightarrow 0 \\
k \rightarrow 0}}\left\|\tau_{j n}\right\|=\lim _{\substack{h \rightarrow 0 \\
k \rightarrow 0}}\left\|-\frac{k^{2}}{6} d_{t}^{3} u_{j n}-\frac{k v h^{2}}{6} \partial_{x}^{4} u_{j n}\right\|=0
\end{gathered}
$$

Therefore we conclude that for $h, k \rightarrow 0$, the truncation error of our proposed scheme is satisfies $\lim \left(\tau_{j n}\right)=0$ and $\lim \left\|\tau_{j n}\right\|=0$. Thus our proposed scheme is consistent with the order of accuracy is $\mathrm{O}\left(h^{2}+k^{2}\right)$. Hence $\left|\tau_{j n}\right| \leq$ $\mathrm{O}\left(h^{2}+k^{2}\right)$.

Theorem 1: If the global error in the proposed scheme is bounded, then the scheme is stable.

Proof: Since the global error is the combination of roundoff error and truncation error in the proposed scheme. Let us consider that $E_{j n}$ is global error and $\varepsilon_{j n}$ round off error in the scheme. And where a round of error defined by $\varepsilon_{j n}=$ $u_{j n}-\tilde{u}_{j n}$ is the error between two numerical solutions. Now substituting this into the proposed scheme the general round 


$$
\begin{aligned}
& D \varepsilon_{j+1 n}=G \varepsilon_{j n+1}+H \varepsilon_{j+1 / 2 n} \\
& \quad \varepsilon_{j+1 n}=D^{-1} G \varepsilon_{j n}+D^{-1} H \varepsilon_{j+1 / 2 n}
\end{aligned}
$$

Thus the global error is

$$
\begin{aligned}
E_{j n} & =\varepsilon_{j+1 n}+\tau_{j n} \\
E_{j+1 n+1} & =D^{-1} G \varepsilon_{j n+1}+D^{-1} H \varepsilon_{j+1 / 2 n}+\tau_{j n}
\end{aligned}
$$

Apply the matrix norm on both sides of Eq.(18) we obtain:

$$
\begin{gathered}
\left\|E_{j+1 n+1}\right\|=\left\|D^{-1} G \varepsilon_{j n}+D^{-1} H \varepsilon_{j+1 / 2 n}+\tau_{j n}\right\| \\
\leq\left\|D^{-1} G \varepsilon_{j n}\right\|+\left\|D^{-1} H \varepsilon_{j+1 / 2 n}\right\|+\left\|\tau_{j n}\right\| \\
\leq C\left\{\frac{1}{\|H\|}|| \varepsilon_{j n}\left\|+\frac{1}{\|G\|}\left|\varepsilon_{j+1 / 2 n}\right|+\frac{1}{\|G H\|}\right\| \tau_{j n} \|\right\} \\
\leq C\left\{\max _{j}\left(\frac{1}{\|H\|}\left|\varepsilon_{j 0}\right|, \frac{1}{\|G\|}\left|\varepsilon_{j+1 / 2} 0\right|\right)+\frac{1}{\|G H\|}\left\|\tau_{j n}\right\|\right\} \leq C\left(h^{2}+k^{2}\right) .
\end{gathered}
$$

where $C=D^{-1} G H$. Hence the global error is bounded so that the proposed scheme is stable.

Corollary 1: Under the condition of the above theorem the proposed scheme is given in Eq. (12) is a well-posed scheme with the given initial and boundary condition.

Proof: Let $u\left(x_{j}, t_{n}\right)$ and $u_{j n}$ are the exact and numerical solution of PDE in Eq.(1) at grid point $\left(x_{j}, t_{n}\right)$. Then the global error at the initial condition $\left(x_{j}, t_{0}\right)$. Is defined by $E_{j, 0}=u\left(x_{j}, t_{0}\right)-u_{j 0}$. By using the condition of the above theorem we have:

$$
\left\|E_{j, 0}\right\|=\left\|u\left(x_{j}, t_{0}\right)-u_{j 0}\right\|=0
$$

Hence the scheme is well-posed at $\left(x_{j}, t_{0}\right)$ for the initial condition. For $j \neq 0$ and $n=1(1) N$, the global error is

$$
\left\|E_{j, n}\right\|=\left\|u\left(x_{j}, t_{j}\right)-u_{j n}\right\| \leq C\left(h^{2}+k^{2}\right)
$$

Hence $\left\|E_{j, n}\right\| \rightarrow 0$ for both $h, k \rightarrow 0$. Thus the scheme is well-posed. So it is convergent.

Criteria for Investigation the Accuracy of Proposed

In this section, we investigate the accuracy of the present method. To show the accuracy of the present method, the Root Mean Square (RMS) error [33] norm $\left(L_{2}\right)$ and maximum absolute error norm $\left(L_{\infty}\right)$ are used to measure the accuracy of the proposed method. The RMS error and maximum absolute error are calculated as follows in the reference [11] given by:

$$
L_{2}=\sqrt{h \sum_{j=1}^{M}\left(\left|U\left(x_{j}, t_{n}\right)-u_{j n}\right|\right)^{2}}
$$

And the maximum absolute error is calculated by:

$$
E_{\infty}=\max _{1 \leq j \leq M}\left|U\left(x_{j}, t_{N}\right)-u_{j N}\right|
$$

Where $U\left(x_{j}, t_{n}\right)$ and $u_{j n}$ are respectively exact and numerical solutions of the Advection-diffusion type equation at the grid point $\left(x_{j}, t_{n}\right)$.

\section{Results and Numerical Experiments}

To test the validity of the proposed method, we have considered the following model problem.

Example1: Consider the in Eq. (1) on $[0,1]$ considered by Soyoon Bak et al. in [1] with the shock initial condition initial and boundary conditions respectively.

$$
u(x, 0)=\left\{\begin{array}{c}
9 x, 0 \leq x \leq 1 / 10 \\
1-x, 1 / 10<x \leq 1
\end{array}, u(0, t)=0=u(1, t)\right.
$$

Example 2: Consider the in Eq. (1) on $[0,1]$ considered by Amit Kumar Verma et. al. in [11] with the periodic initial condition initial and Dirichlet boundary conditions respectively.

$$
\begin{gathered}
u(x, 0)=\sin \pi x \\
u(0, t)=0=u(1, t)
\end{gathered}
$$

The unique exact solution of the above Burger's equation is given by:

$$
U(x, t)=\left[\frac{\sum_{m=1}^{\infty} A_{m} e^{-v m^{2} \pi^{2} t / 2} \sin (m \pi x)}{A_{0}+\sum_{m=1}^{\infty} A_{m} e^{-v m^{2} \pi^{2} t / 2} \cos (m \pi x)}\right]
$$

where $\quad A_{0}=\int_{0}^{1} e^{\left(\frac{1}{\pi v}(\cos (\pi x)-1)\right)} d x \quad$ and $A_{m}=\int_{0}^{1} e^{\left(\frac{1}{\pi v}(\cos (\pi x)-1) \cos (\mathrm{m} \pi \mathrm{x})\right)} d x$

Example3: Consider the in Eq. (1) on $[0,1]$ considered by Amit Kumar Verma et al. in [11]

The unique exact solution of the above Burger's equation is given by:

$$
U(x, t)=\pi v\left[\frac{e^{\left(-v^{2} \pi^{2} t / 4\right)} \sin (\pi x)+4 e^{\left(-v^{2} \pi^{2} t\right)} \sin (2 \pi x)}{4+e^{\left(-v^{2} \pi^{2} t / 4\right)} \cos (\pi x)+2 e^{\left(-v^{2} \pi^{2} t\right)} \cos (2 \pi x)}\right]
$$

Example 4: Consider the in Eq. (1) on $[0,1]$ considered by Amit Kumar Verma et al. in [11] with initial condition initial and Dirichlet boundary conditions respectively. 


$$
\begin{aligned}
& u(x, 0)=4 x(1-x) \\
& u(0, t)=0=u(1, t)
\end{aligned}
$$

The unique exact solution of the above Burger's equation is given by:

$$
\begin{aligned}
& U(x, t)=\pi v\left[\frac{\sum_{m=1}^{\infty} A_{m} e^{-v m^{2} \pi^{2} t / 2} \sin (m \pi x)}{A_{0}+\sum_{m=1}^{\infty} A_{m} e^{-v m^{2} \pi^{2} t / 2} \cos (m \pi x)}\right] \\
& \text { where } \\
& A_{0}=\int_{0}^{1} e^{\left(\frac{2 x^{2}}{3 v}(3-2 x)\right)} d x \\
& A_{m}=2 \int_{0}^{1} e^{\left(\frac{1}{\pi v}(3-2 x) \cos (\mathrm{m} \pi \mathrm{x})\right)} d x
\end{aligned}
$$

Table 1. Root Mean Square (RMS) error norm $\left(L_{2}\right)$ and maximum absolute error norm $\left(L_{\infty}\right)$ for problem give in example one with computations carried out until final time $T=0.1$ with different mesh size and $v=0.01$.

\begin{tabular}{llll}
\hline Mesh size & & By present method & \\
\hline $\mathbf{h}$ & $\mathbf{k}$ & $\boldsymbol{L}_{\infty}$ & $\boldsymbol{L}_{2}$ \\
\hline 0.1 & 0.01 & $3.3321 \mathrm{E}-04$ & $1.0537 \mathrm{E}-04$ \\
0.0125 & 0.01 & $6.2625 \mathrm{E}-06$ & $7.0016 \mathrm{E}-07$ \\
0.001 & 0.0125 & $8.3026 \mathrm{E}-06$ & $2.6255 \mathrm{E}-07$ \\
\hline
\end{tabular}

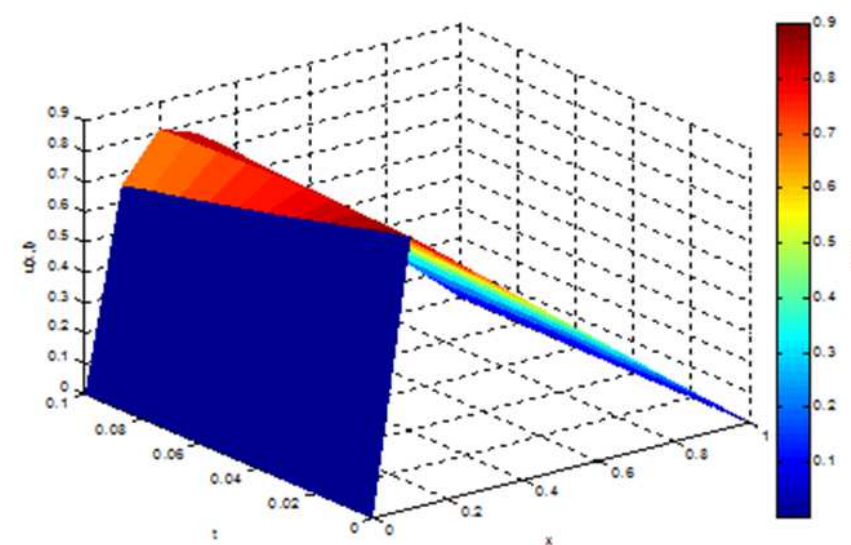

(a)

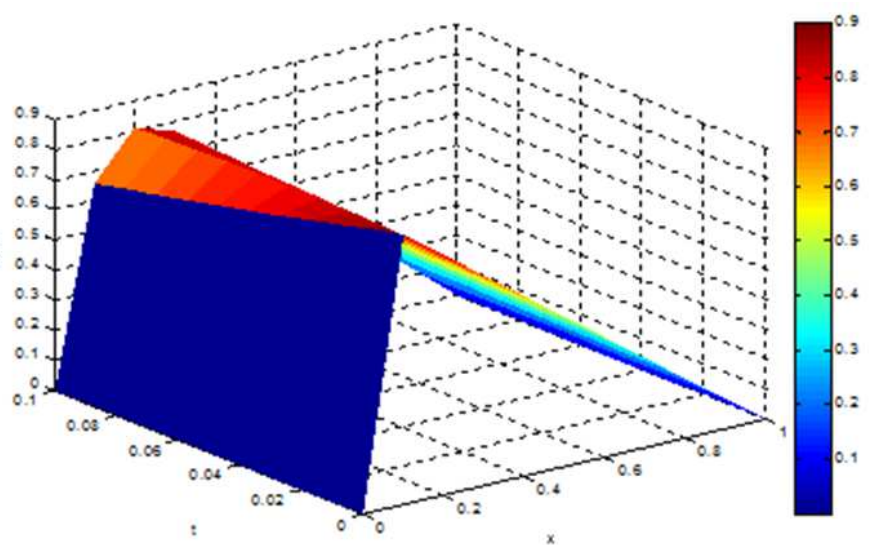

(b)

Figure 1. a) Profile of approximate solution for Example 1 with $h=0.1, k=0.01$ and $v=0.01$; b) Profile of exact solution for Example 1 with $h=0.1$, $k=0.01$ and $v=0.01$.

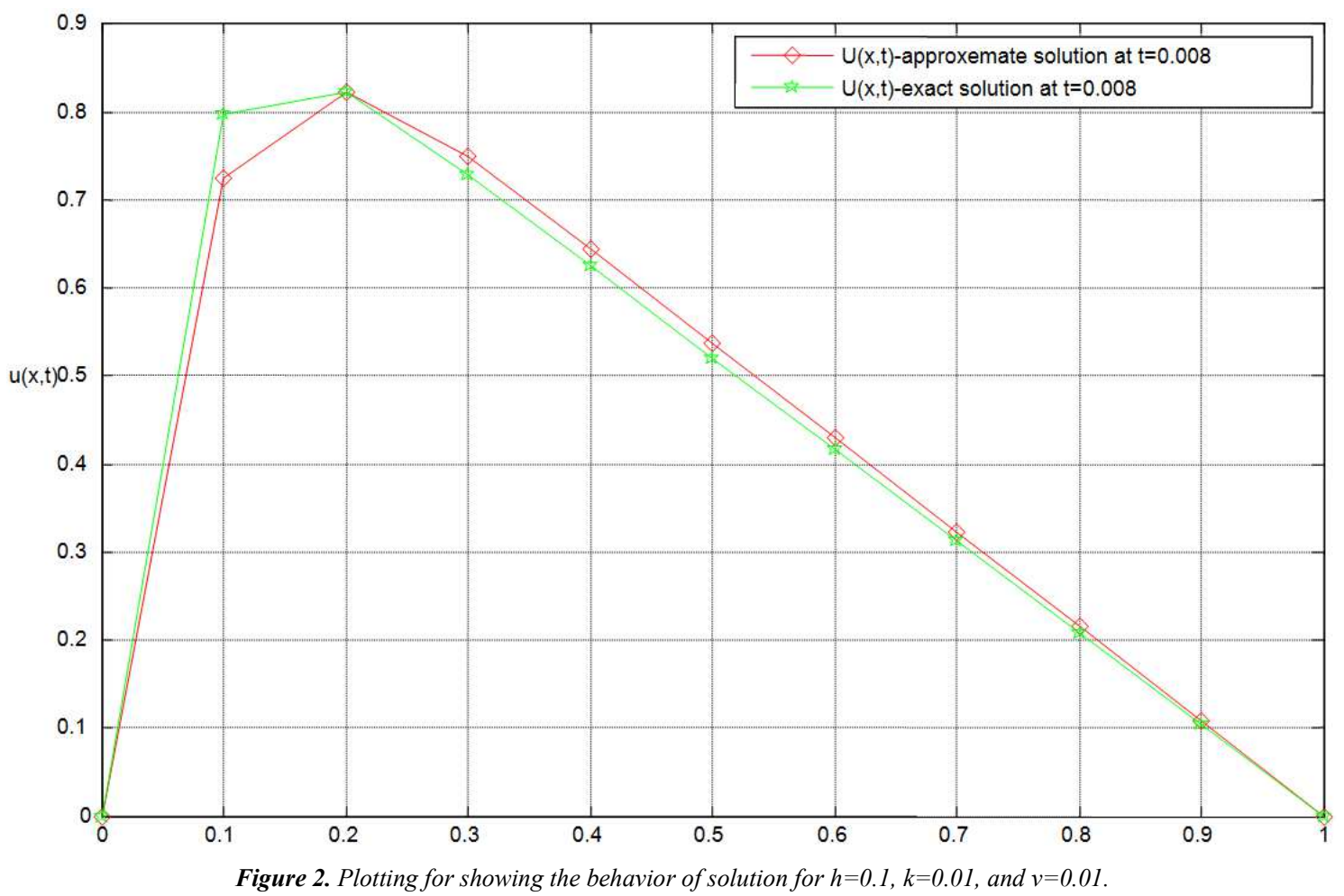




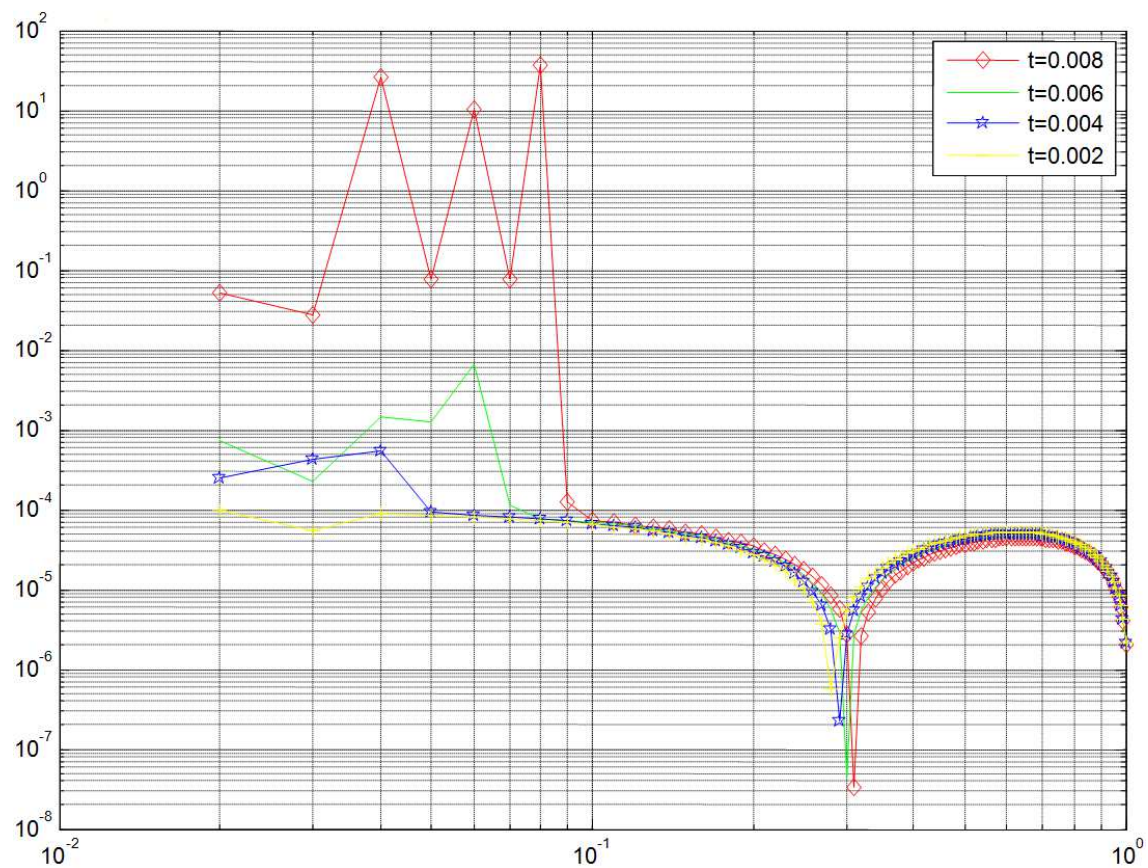

Figure 3. $\log$-log plotting for showing the behavior of error in solution for $h=k=0.01$ and $v=0.01$.

Table 2. Comparison of Root Mean Square (RMS) error norm $\left(L_{2}\right)$ and maximum absolute error norm $\left(L_{\infty}\right)$ for problem give in example two with $v=2$.

\begin{tabular}{|c|c|c|c|c|c|}
\hline \multicolumn{6}{|c|}{ With computations carried out until final time $T=0.001$} \\
\hline \multicolumn{2}{|c|}{ Mesh size } & \multicolumn{2}{|c|}{ By Amit K. V. et al. in [11] } & \multicolumn{2}{|c|}{ By present Method } \\
\hline $\mathrm{h}$ & $\mathrm{k}$ & $L_{\infty}$ & $L_{2}$ & $L_{\infty}$ & $L_{2}$ \\
\hline 0.0125 & 0.0001 & $2.71275 \mathrm{E}-4$ & $6.41526 \mathrm{E}-05$ & $6.4299 \mathrm{E}-07$ & 7.1889E-08 \\
\hline 0.01 & 0.0001 & & & $6.3611 \mathrm{E}-05$ & $6.3611 \mathrm{E}-06$ \\
\hline 0.008 & 0.0001 & & & 4.6723E-03 & 4.1790E-04 \\
\hline \multicolumn{6}{|c|}{ With computations carried out until final time $T=0.01$} \\
\hline $\mathrm{h}$ & $\mathrm{k}$ & $L_{\infty}$ & $L_{2}$ & $L_{\infty}$ & $L_{2}$ \\
\hline 0.0125 & 0.0001 & $2.413 \mathrm{E}-04$ & $5.82562 \mathrm{E}-05$ & 4.5497E-06 & $1.4387 \mathrm{E}-07$ \\
\hline 0.05 & 0.001 & & & $1.2520 \mathrm{E}-05$ & $2.7995 \mathrm{E}-06$ \\
\hline 0.04 & 0.001 & & & $1.4122 \mathrm{E}-04$ & $2.8243 \mathrm{E}-05$ \\
\hline \multicolumn{6}{|c|}{ With computations carried out until final time $T=0.1$} \\
\hline h & $\mathrm{k}$ & $L_{\infty}$ & $L_{2}$ & $L_{\infty}$ & $L_{2}$ \\
\hline 0.0125 & 0.0001 & $9.54852 \mathrm{E}-05$ & $2.27535 \mathrm{E}-05$ & 7.6714E-07 & $2.4259 \mathrm{E}-07$ \\
\hline 0.04 & 0.0001 & & & 1.3057E-07 & 2.6114E-08 \\
\hline 0.05 & 0.0001 & & & $2.0261 \mathrm{E}-07$ & 4.5305E-08 \\
\hline
\end{tabular}

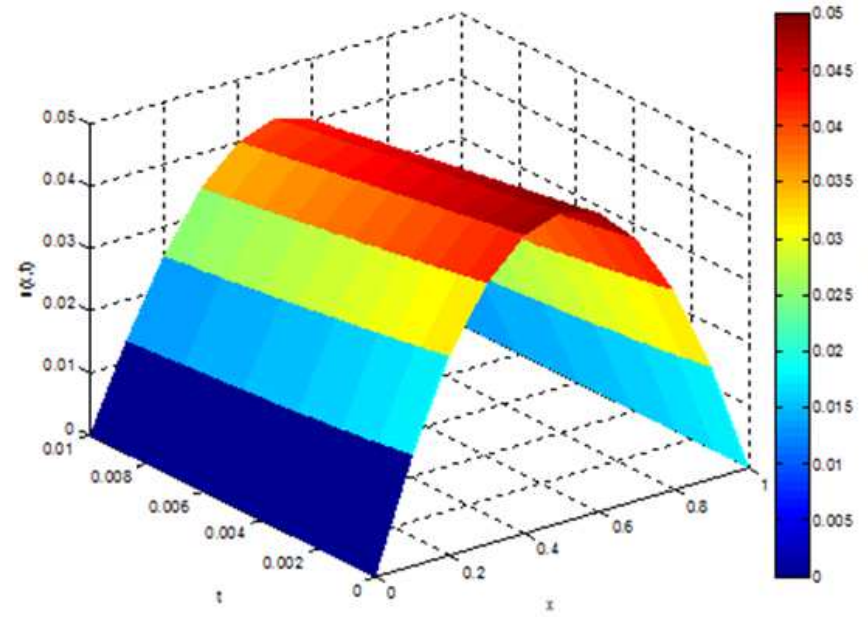

(a)

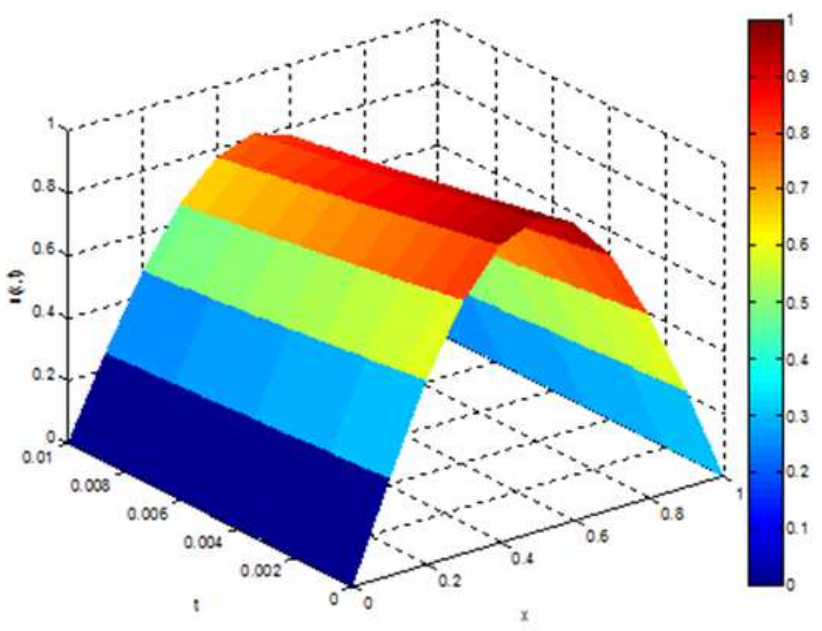

(b)

Figure 4. a) Profile graph of approximate solution for Example 2 with $h=0.1, k=0.0001$, and $v=2$; b) Profile graph of the exact solution for Example 2 with $h=0.1, k=0.0001$, and $v=2$. 


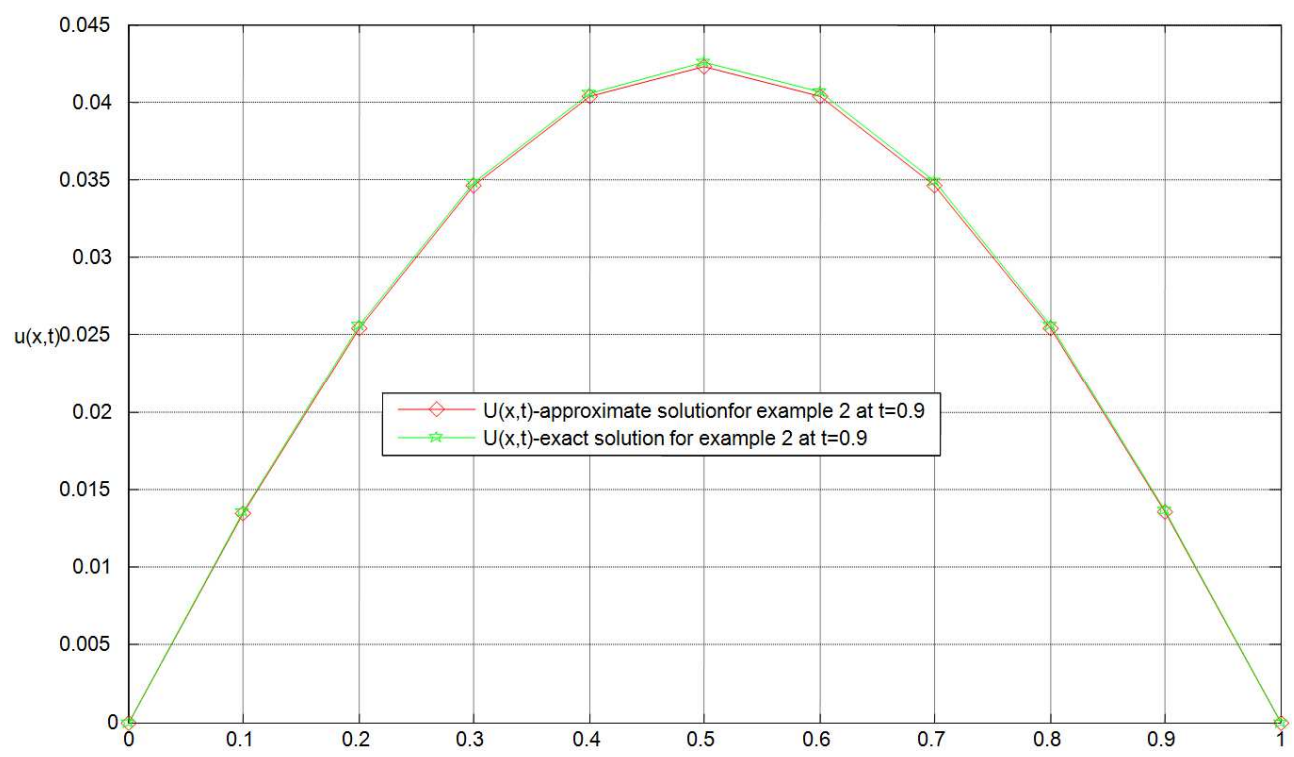

Figure 5. Plotting graph of Example 2 for showing the behavior of solution for $h=0.1, k=0.0001$, and $v=2$.

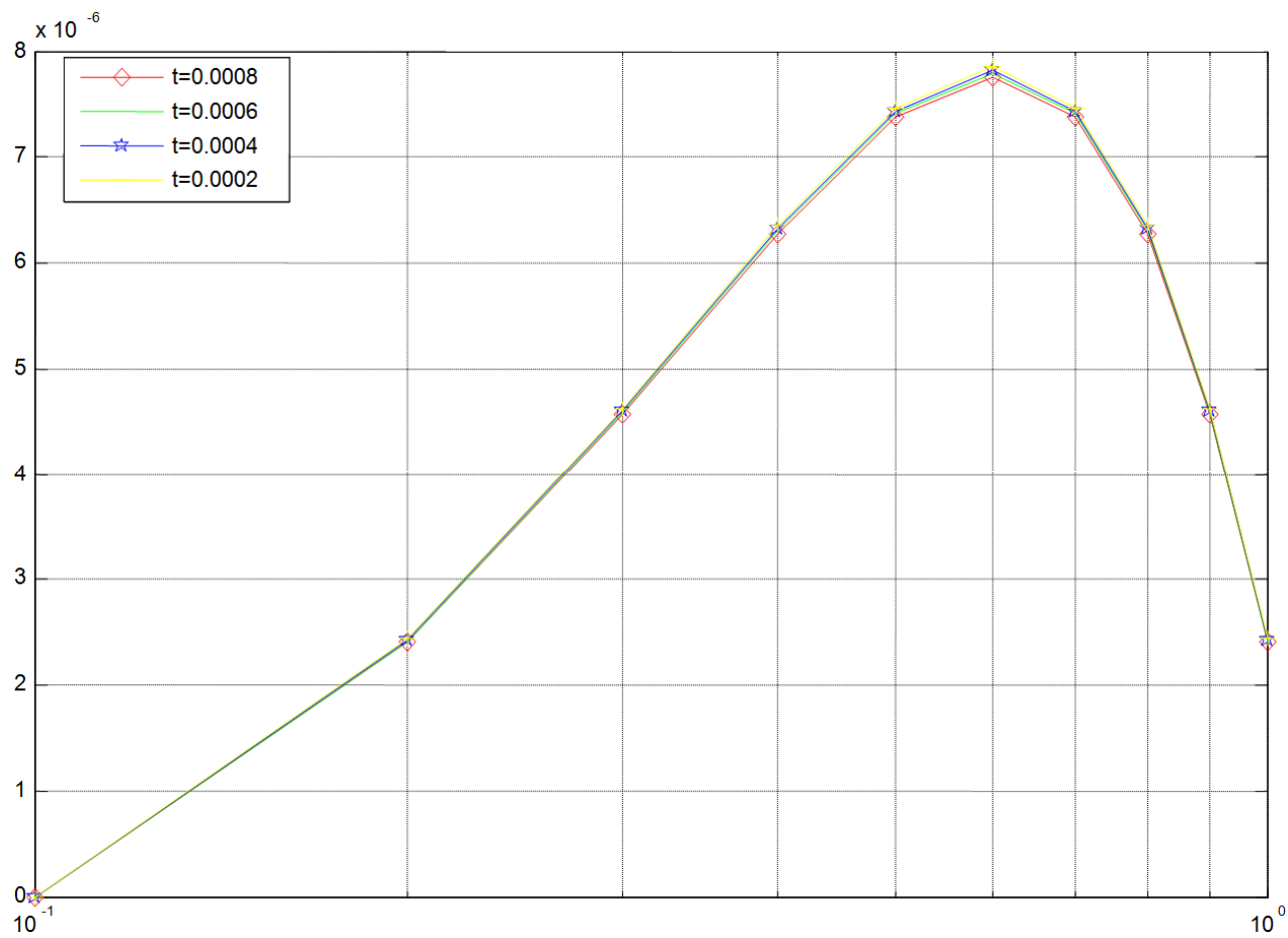

Figure 6. Semilogx plotting for showing the behavior of error in solution for $h=0.1, k=0.0001$, and $v=2$.

Table 3. Comparison of Root Mean Square (RMS) error norm $\left(L_{2}\right)$ and maximum absolute error norm $\left(L_{\infty}\right)$ for problem give in example three with $v=0.002$.

\begin{tabular}{|c|c|c|c|c|c|c|c|}
\hline \multicolumn{2}{|l|}{ Mesh size } & \multicolumn{2}{|c|}{ By SS Xie et al [29] } & \multicolumn{2}{|c|}{ By Amit et al. in[11] } & \multicolumn{2}{|c|}{ By present method } \\
\hline \multicolumn{8}{|c|}{ With computations carried out until final time $T=1.7$} \\
\hline $\mathrm{h}$ & $\mathrm{k}$ & $L_{\infty}$ & $L_{2}$ & $L_{\infty}$ & $L_{2}$ & $L_{\infty}$ & $L_{2}$ \\
\hline 0.0005 & 0.01 & 2.9704E-02 & $3.5936 \mathrm{E}-03$ & $5.02 \mathrm{E}-04$ & $1.67 \mathrm{E}-05$ & $5.264 \mathrm{E}-06$ & $1.177 \mathrm{E}-06$ \\
\hline 0.1 & 0.0001 & & & & & $2.474 \mathrm{E}-05$ & 7.824E-06 \\
\hline \multicolumn{8}{|c|}{ With computations carried out until final time $T=3$} \\
\hline $\begin{array}{l}0.0005 \\
0.1\end{array}$ & $\begin{array}{l}0.01 \\
0.0001\end{array}$ & $1.90097 \mathrm{E}-02$ & $2.635 \mathrm{E}-03$ & $2.1289 \mathrm{E}-4$ & $8.14 \mathrm{E}-05$ & $\begin{array}{l}5.933 \mathrm{E}-05 \\
3.062 \mathrm{E}-05\end{array}$ & $\begin{array}{l}1.326 \mathrm{E}-05 \\
9.685 \mathrm{E}-06\end{array}$ \\
\hline \multicolumn{8}{|c|}{ With computations carried out until final time $T=3.5$} \\
\hline $\begin{array}{l}0.0005 \\
0.01\end{array}$ & $\begin{array}{l}0.01 \\
0.001\end{array}$ & $1.67887 \mathrm{E}-02$ & $2.417 \mathrm{E}-03$ & $1.687 \mathrm{E}-04$ & $6.69 \mathrm{E}-5$ & $\begin{array}{l}7.186 \mathrm{E}-05 \\
1.9176 \mathrm{E}-05\end{array}$ & $\begin{array}{l}1.607 \mathrm{E}-05 \\
6.064 \mathrm{E}-06\end{array}$ \\
\hline
\end{tabular}




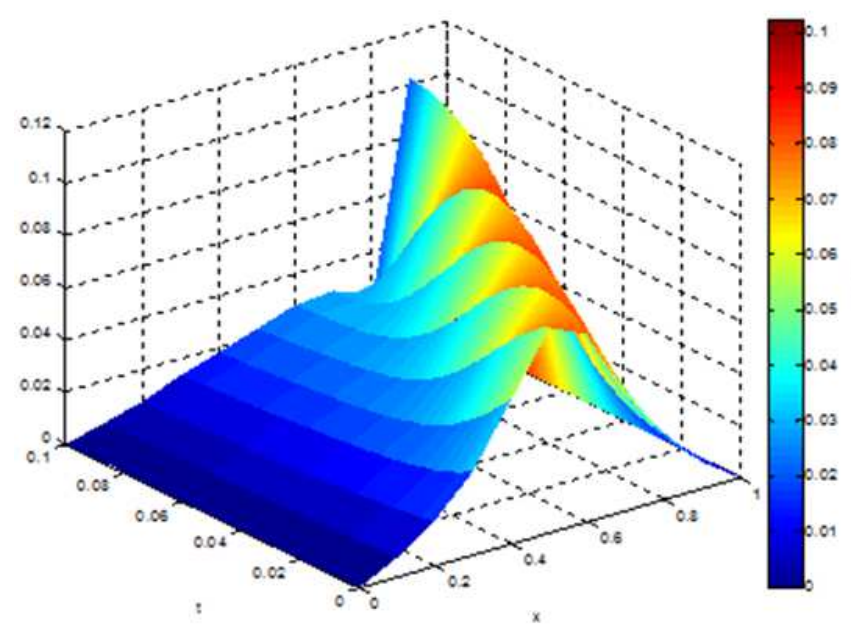

(a)

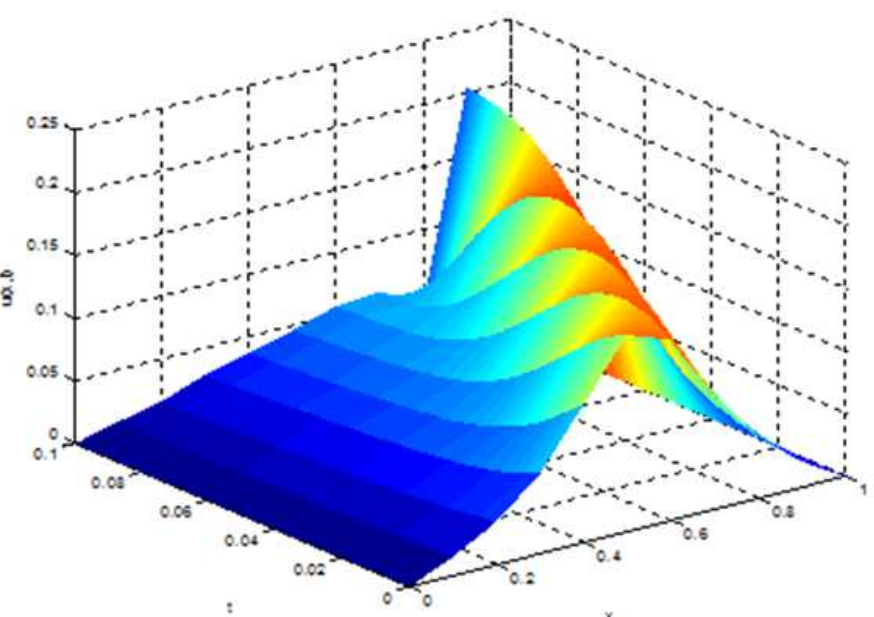

(b)

Figure 7. a) Profile graph of approximate solution for Example 3 with $h=0.05, k=0.01$, and v=0.002; b) Profile graph of Exact solution for Example 3 with $h=0.05, k=0.01$, and $v=0.002$.

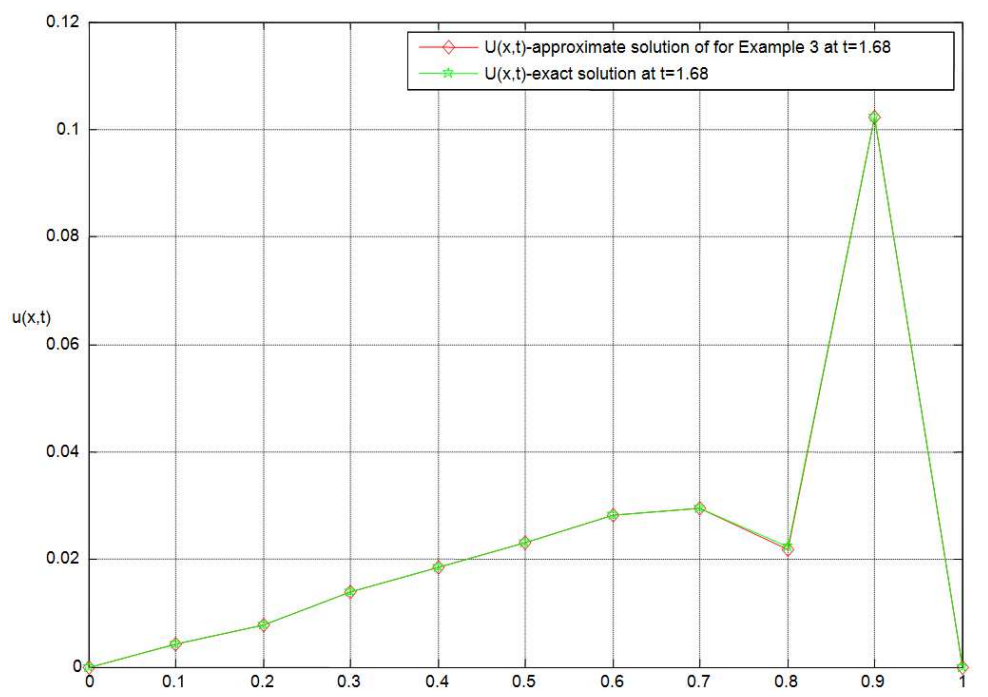

Figure 8. Plotting graph of Example 3 for showing the behavior of solution with $h=0.05, k=0.01$, and $v=0.002$.

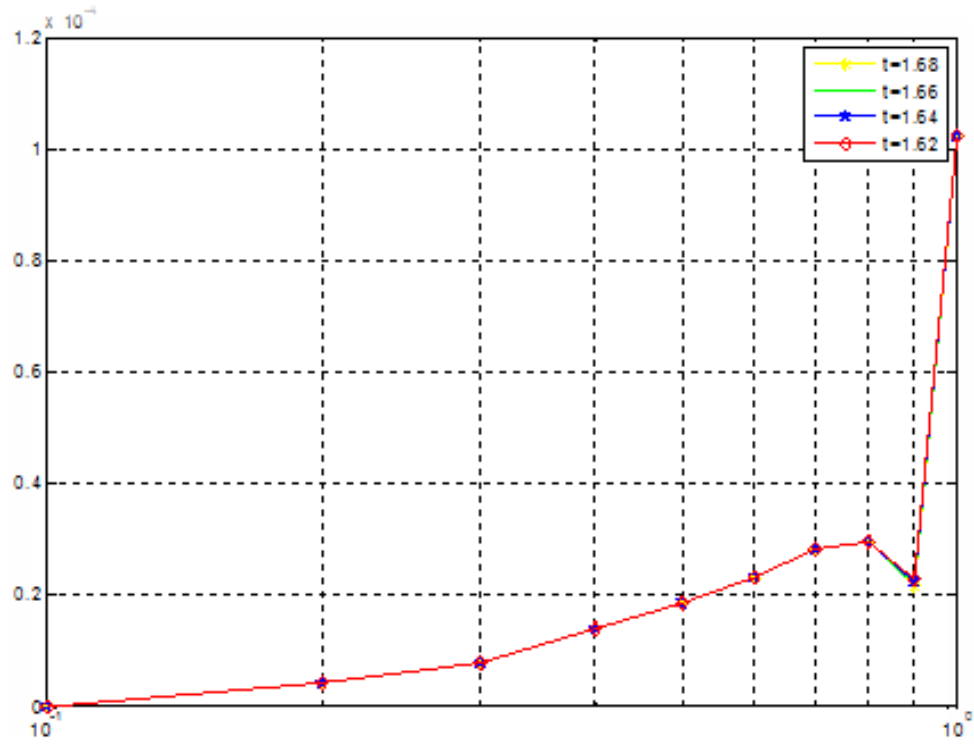

Figure 9. Semi log plotting for showing the behavior of error in solution for $h=0.05, k=0.01$, and $v=0.002$. 
Table 4. Comparison of Root Mean Square $(R M S)$ error norm $\left(L_{2}\right)$ and maximum absolute error norm $\left(L_{\infty}\right)$ for problem give in example four with $v=2$.

\begin{tabular}{|c|c|c|c|c|c|}
\hline \multicolumn{2}{|c|}{ Mesh-size } & \multicolumn{2}{|c|}{ By Amit et al. in[11] } & \multicolumn{2}{|c|}{ By present method } \\
\hline h & $\mathbf{k}$ & $\boldsymbol{L}_{\infty}$ & $L_{2}$ & $\boldsymbol{L}_{\infty}$ & $L_{2}$ \\
\hline \multicolumn{6}{|c|}{ With computations carried out until final time $T=0.001$} \\
\hline 0.0125 & 0.0001 & $2.64275 \mathrm{E}-04$ & $6.55334 \mathrm{E}-05$ & $5.5124 \mathrm{E}-07$ & $6.1630 \mathrm{E}-08$ \\
\hline 0.02 & 0.0001 & & & 2.7535E-09 & 3.8941E-10 \\
\hline \multicolumn{6}{|c|}{ With computations carried out until final time $T=0.01$} \\
\hline 0.0125 & 0.0001 & 2.35909E-04 & $6.07706 \mathrm{E}-05$ & $1.8785 \mathrm{E}-07$ & $2.6050 \mathrm{E}-08$ \\
\hline 0.05 & 0.0001 & & & 2.6863E-07 & 4.9883E-08 \\
\hline \multicolumn{6}{|c|}{ With computations carried out until final time $T=0.1$} \\
\hline 0.0125 & 0.0001 & $9.85169 \mathrm{E}-05$ & $2.46429 \mathrm{E}-05$ & $9.3345 \mathrm{E}-08$ & $1.3201 \mathrm{E}-08$ \\
\hline 0.04 & 0.0001 & & & 4.0702E-07 & 8.1404E-08 \\
\hline
\end{tabular}

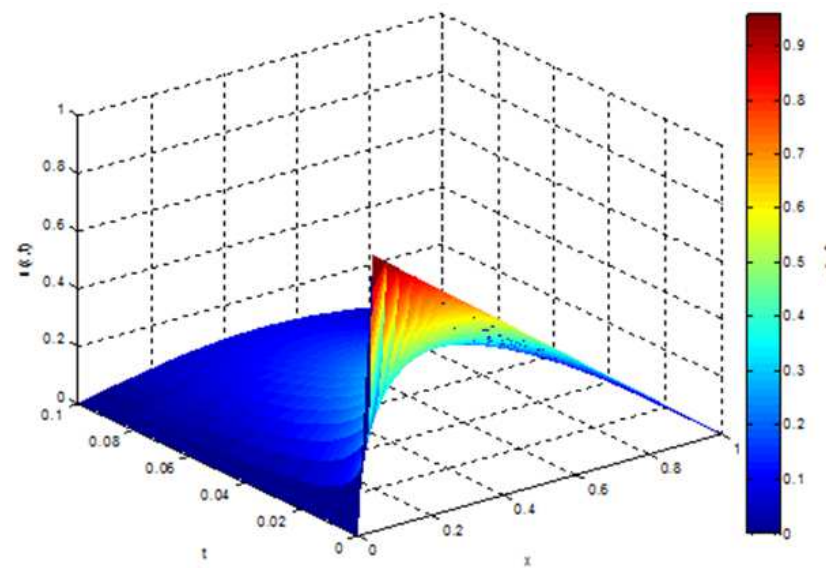

(a)

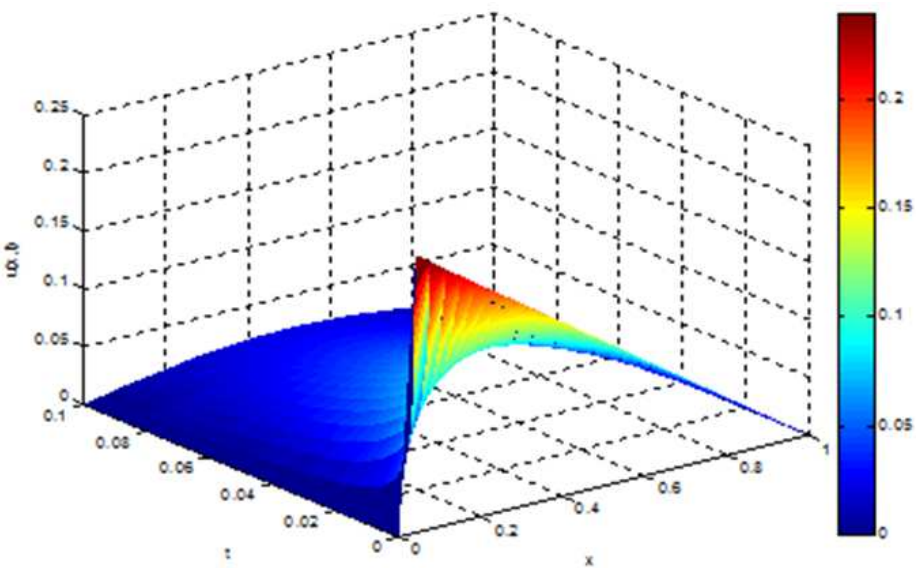

(b)

Figure 10. a) Profile graph of approximate solution for Example 4 with $h=0.01, k=0.01$, and $v=2$; b) Profile graph of exact solution for Example 4 with $h=0.01, k=0.01$ and $\nu=2$.

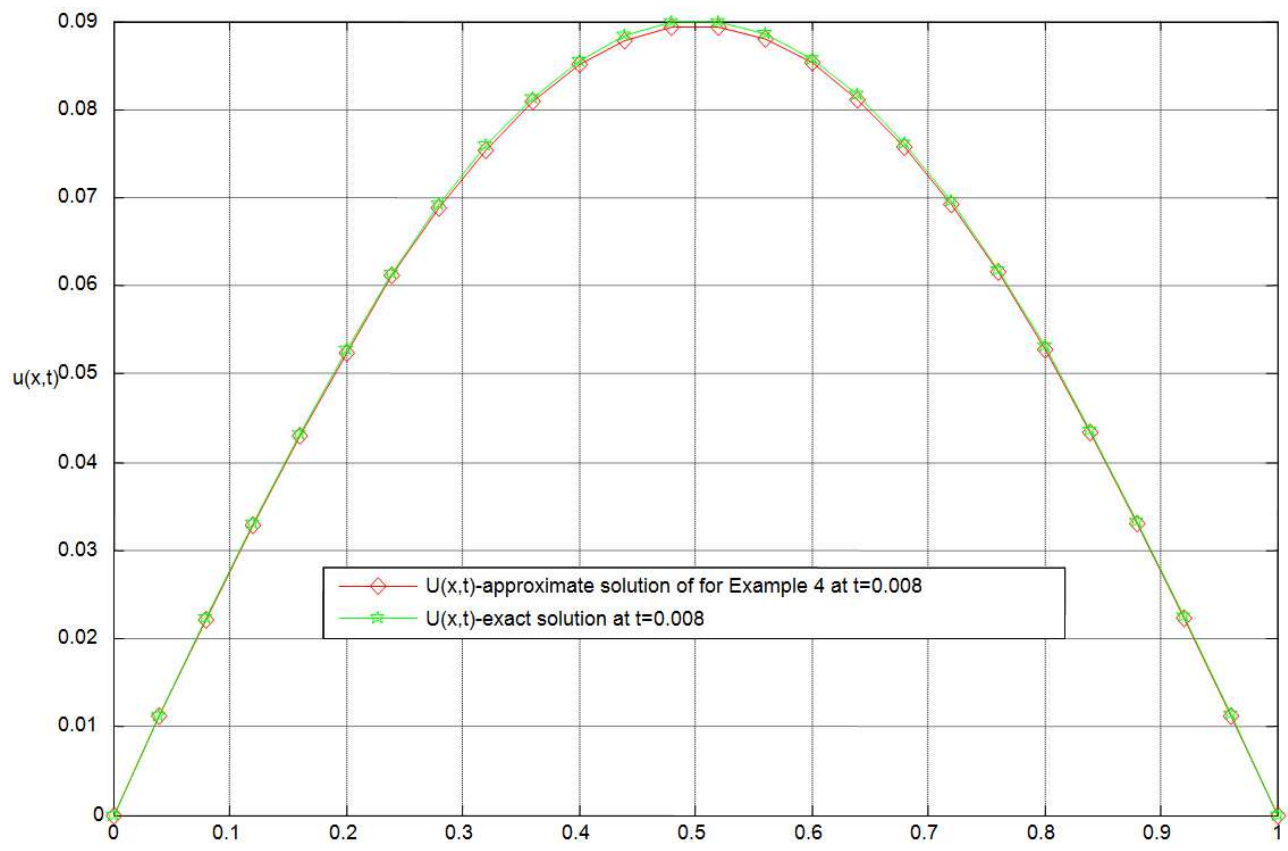

Figure 11. Plotting graph of Example 4 for showing the behavior of solution with $h=0.1, k=0.01$, and $v=2$. 


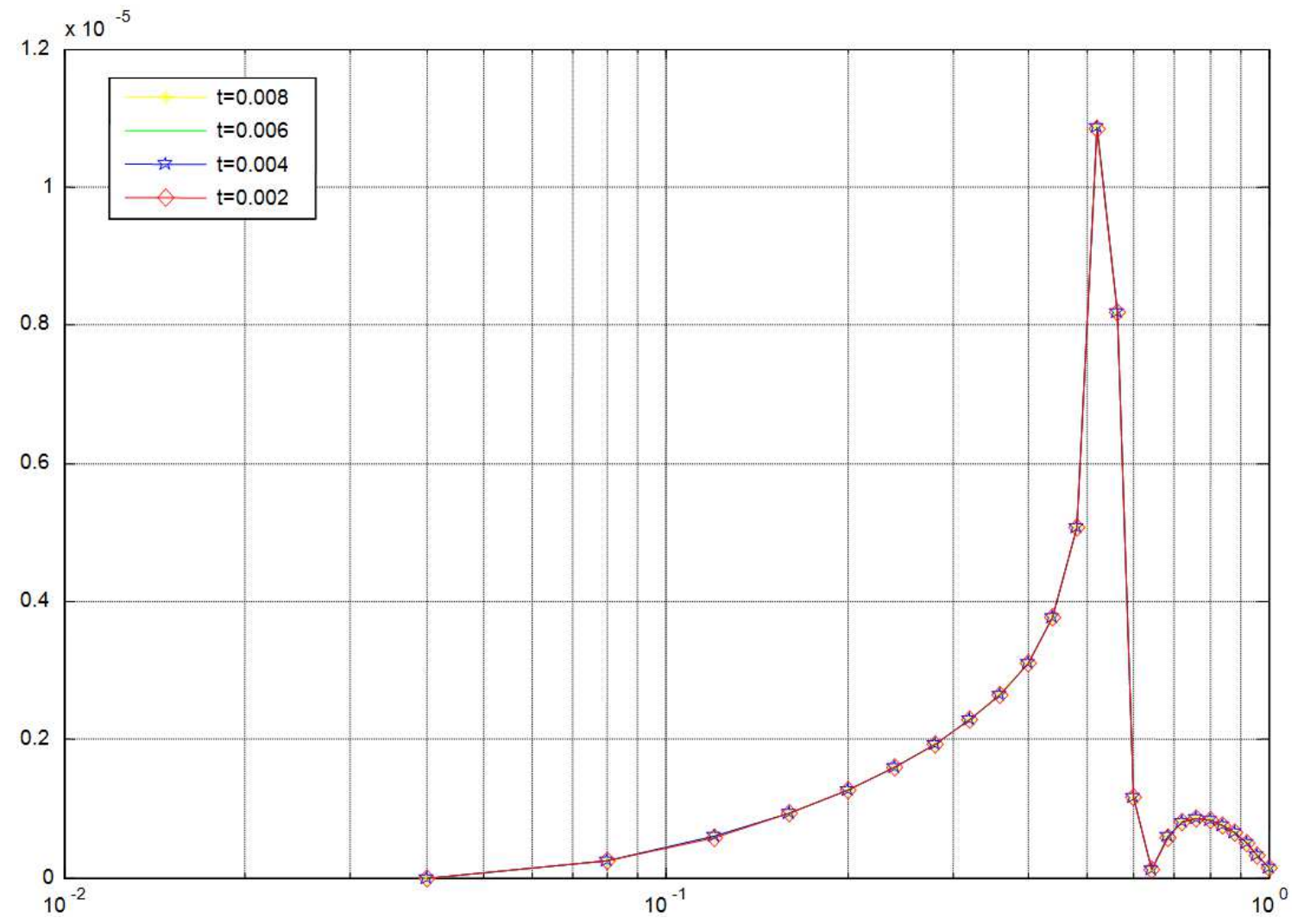

Figure 12. Semilogx plotting for showing the behavior of error in solution for Example 4 with $h=0.1, k=0.01$, and $v=2$.

\section{Discussion}

In this paper, the piece-wise parabolic method is presented for solving the one-dimensional advection-diffusion type equation and application to the burger equation. To demonstrate the competence of the method, four model examples are solved by taking different values for step size $\mathrm{h}$, and time step $\mathrm{k}$. Numerical results obtained by the present method have been associated with numerical results obtained by the method in $[1,11,29]$ and the results are summarized in Tables and graphs. Moreover, in the present numerical computation, the result presented in Table 1-4 show that the root mean square error norm $\left(L_{2}\right)$ and maximum absolute error norm $\left(L_{\infty}\right)$ decrease rapidly as the number of mesh points $\mathrm{M}$ increases in a spatial variable with fixed $\mathrm{N}$ in the temporal direction. In this case, the accuracy of the present method decrees. Further, as shows in Figures 1a-1b, 2, 4a-4b, 5, 7a-7b, 8, 10a-10b, and 11 the proposed method approximates the exact solution very well for different values of step length $h$ and time step $k$. To further verify the applicability of the planned method, graphs were plotted aimed at Examples 1, 2, 3, and 4 for exact solutions versus the numerical solutions obtained by the present method showed by figures $2,5,8$, and 11 respectively indicate good agreement of the results, and proves the reliability of the present method. Also, Figures 3, 6, 9, and 12 respectively specify the behavior of absolute error obtained by the present method within the effects of mesh sizes on the solution domain. Hence, the numerical results presented in this paper validate the improvement of the proposed method over some of the existing methods described in the literature. Both the theoretical and numerical error bounds have been established. The results in the Tables are further confirmed that the computational rate of convergence and theoretical estimates are in agreement.

Comparison among Table 2-Table 4 and the graphs of the numerical and exact solution of one-dimensional advectiondiffusion type equation shows that the present method generates a more accurate result and it is superior to the method developed in $[1,11,29]$ and It is approximate the exact solution very well.

\section{Conclusion}

A new approach, the piece-wise parabolic method is using to solve the one-dimensional advection-diffusion type equation, and application to burger equation numerically is presented in this study. The comparison of the results obtained by the present method with other methods reveals that the present method is more convenient, reliable, and effective. An error analysis based on the Fourier series is also developed in this study. As it can be seen that, the accuracy improves when $M$ small grid number in spatial direction with fixed $N$ in the temporal direction. In a summary, the piecewise parabolic method is a reliable method that is capable to solve the one-dimensional advection-diffusion type equation and burger equation. Based on the findings, this method is 
well approximate and gives better accuracy of the numerical solution with a fixed time step, $k$, and large step size $h$.

\section{Acknowledgments}

First of all, I would like to express my thanks to Allah, for giving me life and time to accomplish my tasks. In the name of Allah, The Most Greatest and Most Merciful, there is no power except by the power of Allah and I humbly return my acknowledgment that all knowledge belongs to Allah. I thank Allah for granting me this opportunity to broaden my knowledge in this field. Nothing is possible unless He made it is possible.

Secondly, greatly appreciate the help of my mother Shamo Busho, and my father Aliyi Koroche, without the help them I would not be here today.

Last but not least, thanks a lot to all my beloved friends; your kindness and helps will be a great memory for me.

\section{References}

[1] Soyoon Bak, Philsu Kim, Xiangfan Piao, and Sunyoung Bu. Numerical solution of advection- Diffusion type equation by modified error correction scheme. Advances in Difference Equations, 2018.

[2] Ram Jiwari, R. C. Mittal, Kapil K. Sharma. A numerical scheme based on the weighted average differential quadrature method for the numerical solution of Burgers' equation. Applied Mathematics and Computation 219 (2013) 6680-6691.

[3] E. N. Aksan, Quadratic B-spline finite element method for the numerical solution of the Burgers' Equation, Appl. Math. Comput. 174 (2006) 884-896.

[4] H. Bateman, Some recent researches on the motion of fluids, Mon. Weather Rev. 43 (1915) 163-170.

[5] J. M. Burgers, A mathematical model illustrating the theory of turbulence, Adv. Appl. Mech., vol. I, Academic Press, New York, 1948. 171-199.

[6] Ram Tiwari. A Haar wavelet quasilinearization approach for numerical simulation of Burgers' Equation. Computer Physics Communications 183 (2012) 2413-2423.

[7] J. M. Burgers, Mathematical example illustrating relations occurring in the theory of turbulent fluid motion, Trans. Roy. Neth. Acad. Sci. Amsterdam 17 (1939) 1-53.

[8] S. Kutluay, A. Esen, A lumped Galerkin method for solving the Burgers equation, Int. J. Comput. Math. 81 (11) (2004) 1433-1444.

[9] Wang, Fajie, Chia-Ming Fan, Chuanzeng Zhang, and Ji Lin. "A localized space-time method of fundamental solutions for diffusion and convection-diffusion problems." Adv. Appl. Math. Mech 12, no. 4 (2020): 940-958.

[10] Sachin S. Wani and Sarita H. Thakar. Crank-Nicolson type method for burgers equation. International Journal of Applied Physics and Mathematics, Vol. 3, No. 5, September 2013.

[11] Amit Kumar Verma, Mukesh Kumar Rawani, Ravi P. Agarwal. On a seventh order convergent weakly $L$-stable Newton Cotes formula with application on Burger's equation. 2019.

[12] Khater, A. H., Temsah, R. S., and Callebaut, D. K. Numerical solutions for some coupled nonlinear evolution equations by using the spectral collocation method. Mathematical and Computer Modeling, 48 (7-8), 2008, 1237-1253.

[13] S. Abbasbandy, M. T. Darvishi, A numerical solution of Burgers' equation by modified Adomian method, Appl. Math. Comput. 163, 2005, 1265-1272.

[14] J. M. Burgers, Application of a model system to illustrate some points of the statistical theory of free turbulence, Proc. R. Nether. Acad. Sci. Amsterdam. 43, 1940, 2-12.

[15] Sirendaoreji, Exact solutions of the two-dimensional Burgers equation, J. Phys. A: Math. Gen. 32, 1999, 6897-6900.

[16] Gowrisankar, S., and Natesan, S. An efficient robust numerical method for singularly Perturbed Burgers' equation. Applied Mathematics and Computation 346, 2019, 385-398.

[17] Reza Abazaria, A. Borhanifar. Numerical study of the solution of the Burgers and coupled Burgers equations by a differential transformation method. Computers and Mathematics with Applications 59, 2010, 2711-2722.

[18] J. G. Zheng, T. S. Lee, and S. H. Winoto. A piecewise parabolic method for barotropic and non Barotropic two-fluid flows. International Journal of Numerical Methods for Heat \& Fluid Flow 18 (6), 2008, 708-729.

[19] A. Mignone, T. Plewa and G. Bodo. The piecewise parabolic method for multidimensional Relativistic fluid dynamics, 2005.

[20] Coleslaw, P. and Woodward, P. R., "The numerical simulation of two-dimensional fluid flow with strong shocks", J. Comput. Phys., Vol. 54, 1984b, 115-73.

[21] Kedir Aliyi Koroche. Weighted Average Based Differential Quadrature Method for One-Dimensional Homogeneous First Order Nonlinear Parabolic Partial Differential Equation. Indian Journal of Advanced Mathematics (IJAM), 1 (1), April 2021.

[22] Coleslaw, P. and Woodward, P. R. (1984b), "The numerical simulation of two-dimensional fluid flow with strong shocks", J. Comput. Phys., Vol. 54, pp. 115-73.

[23] Kedir Aliyi Koroche. Numerical Solution for One Dimensional Linear Types of Parabolic Partial Differential Equation and Application to Heat Equation. Mathematics and Computer Science. 2020; 5: 76-85.

[24] M. W. Oday. Stability and Convergence for nonlinear partial differential equations, Master of Science in Mathematics thesis, Boise State University. 2012.

[25] Rashidinia, J., F. Esfahani, and S. Jamalzadeh. "B-spline collocation approach for the solution of Klein-Gordon equation." 2013: 25-33.

[26] Shokofeh S. and Rashidinia J. Numerical solution of the hyperbolic telegraph the equation by cubic B-spline collocation method. Applied Mathematics and Computation 281, 2016, 28-38.

[27] Smith, Gordon D., Gordon D. Smith, and Gordon Dennis Smith. Numerical solution of partial differential equations: finite difference methods. Oxford university press, 1985. 
[28] Thomas, James William. A numerical method for partial differential equations: finite difference methods. Vol. 22. Springer Science \& Business Media, 2013.

[29] SS Xie, S. Heo, S. Kim, G. Woo, and S. Yi, Numerical solution of one-dimensional Burgers equation using reproducing kernel function, Journal of Computational and Applied Mathematics, 214 (2), 2008, 417-434.

[30] Vijitha Mukundan, Ashish Awasthi. "Numerical Treatment of the Modified Burgers' Equation via backward Differentiation Formulas of Orders Two and Three", International Journal of Nonlinear Sciences and Numerical Simulation, 2018.
[31] International Journal of Numerical Methods for Heat \& Fluid Flow, Volume 22, Issue 7 (2012-09-22).

[32] Tahir Nazir, Muhammad Abbas, Muhammad Yaseen. "Numerical solution of second-order hyperbolic telegraph equation via new cubic trigonometric B-splines approach", Cogent Mathematics, 2017.

[33] Feng Gao, Chunmei Chi. "Numerical solution of nonlinear Burgers' equation using high accuracy multi-quadric quasiinterpolation", Applied Mathematics and Computation, 2014. 